\title{
The Study of Authentic Environment in Authentic Assessment in Developing College Students' English Reading Ability
}

\author{
Lu Shan and Yang Lu
}

Foreign Language College, Guangxi University of Science and Technology, Liuzhou, Guangxi, China (lzlushan@ 163.com)

\begin{abstract}
In order to improve college students' English reading ability, an authentic environment is proposed to be built for the teaching based on authentic assessment. Firstly, based on the authentic assessment theory, the question of how to carry out reform in teaching in the process of developing college students' English reading ability is discussed. Then, taking the communication in daily reading into consideration, a method is proposed to build the authentic assessment environment. Finally, some problems worthy of attention are put forward. Building authentic environment according to the real environment in daily reading will provide foundation for carrying out authentic assessment in developing college students' English reading ability, thus improving the quality of teaching.
\end{abstract}

Keywords-English reading ability, authentic assessment, authentic environment

\section{大学生英语阅读能力培养真实性评价中真实性环境的 研究}

$$
\text { 卢珊 杨露 }
$$

广西科技大学外国语学院, 柳州, 广西, 中国

摘＼cjkstart要＼cjkstart针对大学生英语阅读能力培养，提出构建真实性环境进行基于真实性评价的教学。首先基于真实性评价理论讨论了在大 学生英语阅读能力培养中如何进行教学改革, 其次结合日常阅读中相互交流的情况提出了构建真实性评价环境的方法, 最后结合教学 实践提出了值得注意的几个问题。结合日常阅读的真实环境进行真实性环境的构建，这将为在英语阅读能力培养的教学中开展真实性 评价提供基础, 从而提高教学质量。

关键词 英语阅读能力, 真实性评价, 真实性环境

\section{1. 引言}

培养大学生的英语阅读能力达到应用英语进行交际的 水平, 这一直是大学英语教学的主要目标之一。为此, 如 何提高大学生英语阅读能力的培养效果一直以来都受到专 家和学者们的关注 ${ }^{[1][2]}$ 。已有研究通过引入新的理论和方 法, 从不同的角度出发, 所探讨的方法在一定程度上提高 了大学生的英语阅读能力。但是, 值得注意的是学生在毕 业后的工作生活中使用英语进行交际的能力一直不让人满 意, 因此在大学生英语阅读能力培养中, 阅读能力培养与

2013 年度广西高等教育教学改革工程项目支持 (资助号: 2013JGB202)
真实环境的统一逐渐成为一个研究方 向 $^{[3][4]}$ 。

在教学理论中, 真实性评价作为强调培养过程与真实 环境相统一的理论备受关注。有关真实性评价的研究都把 真实性评价看做 “是与人们在工作中所面临的真实问题相 类似的任务” [5]。因此, 引入真实性评价进行大学生英语 阅读能力培养的教学改革成为提高教学质量的一个有效途 径。而在真实性评价中真实性环境的构建是一个极为重要 的因素, 也是开展真实性评价的基础。

2. 基于真实性评价的大学生英语阅读教学改革 在大学生英语阅读能力培养中进行真实性评价, 就是 
参考学生日后毕业工作生活中可能面对的真实情况进行学 生阅读能力的培养。这主要体现在, 培养过程中采用真实 性的评价环境、真实性的评价标准、真实性的评价过程、 真实性的评价内容。这是与现有的阅读能力培养完全不相 同的方式。

以进行飞机场登机为例。在现有的教学中, 一般是给 出一段文字, 然后提出几个相关的问题让学生进行回答。 教师在教学中, 主要是对给出的文字信息进行逐句的讲解 分析。最后的考核一般以最后几个问题回答是否正确作为 评价标准。评价的主要内容就是学生对问题所涉及的内容 是否按出题人的想法进行理解。这样的考核, 取得高分的 学生并没有体现出较高的英语阅读能力, 在机场面对实际 英语阅读环境也有可能出现问题。

在真实性评价中, 首先将机场可能出现的英语文本提 供给学生。而评价的标准是学生是否能够根据这些文字信 息完成登机, 比如具体从哪个登机口登机, 从哪进行安 检……而评价的过程, 允许学生按照登机的真实过程中应 用英语进行必要的询问。而评价的内容, 除了正常的登机 过程外, 还必须加入飞机延误等常见情况, 让学生进行判 断。这样的阅读训练直接以学生是否能够在真实环境中阅 读英语文本, 获取信息为评判标准, 尽量真实模拟真实环 境进行训练、评价。

显然真实性评价中由于训练过程和评价标准等更接近 实际情况将更有助于学生阅读能力的提高。但是, 在这一 过程中对于真实环境的模拟就成为了关键。一个尽量真实 的语言环境将是开展真实性评价的基础。

\section{3. 真实性评价中真实性环境的构建}

在大学生英语阅读能力真实性评价中, 所谓的真实性 环境主要是语言环境, 体现为提供给学生的英语语言信息 与今后工作生活中真实情况尽量一致, 这既包括文字信息 也包括声音信息。因此, 在真实性环境的构建中可以考虑 从文本信息的提供、信息交流的模拟和任务的设计三个方 面进行。

\section{1 英语文本信息的提供}

在英语阅读能力的真实性评价中, 英语文本是指用英 文提供的信息文本, 既包括文字文本也包括语音文本。如 在超市购物的环境模拟中, 超市中物品的标示、路标等文 字是英语文本，超市内的英语广播也属于文本信息。

在这些文本信息的提供中不能如以往的阅读训练那样 统一以文字的形式, 以一篇短文的形式提供给学生。应该 尽量接近真实环境, 借助现有的教学辅助手段, 通过影像、 视频、图像等多媒体的手段将相关的英文文本信息提供给
学生。

有观点认为这样提供的信息会让学生无法及时、完全 的接受信息, 从而由于遗漏关键信息而产生错误。实际上, 这正是现有英语阅读能力培养的弱点所在。在教学实践中, 就有类似的情况出现。由于长期接受文字信息的阅读训练, 与声音信息结合不足, 当面对相关视频构建的语言环境时 学生不由自主的首先关注文字信息而忽视语言信息。这将 极大的影响学生在真实环境中接受英语信息, 以及应用英 语进行交际和交流。

\section{2 对于信息交流的模拟}

在真实环境中, 人们获得一段文字信息后一般都要进 行一定的交流, 而传统的阅读训练将这一步骤完全省略了。 在传统的英语阅读能力培养中, 学生被要求阅读一段文字 并独立的做出判断, 这在现实情况中是极为少见的。现实 中更常见的情况是阅读一段文字然后与他人就相关问题进 行讨论。在讨论中, 通过引用、分析已有的这段文字与别 人讨论验证、修正自己的观点, 从而实现与他人的交流。

通过交流进行英文阅读这种形式在专业英语的学习中 中更为明显。比如在工科学校, 当学生进入高年级的时候 需要接触一些进口的仪器设备, 或者仅有英文说明书的装 置。调查发现, 在学生间进行一定的交流后, 传统英语阅 读能力测试取得较好成绩的学生在阅读相关英文资料方面 并没有明显优势, 而在学习使用相关仪器设备中更是毫无 优势可言。

在过去的教学中, 有观点认为学生专业英语阅读能力 与通用英语阅读能力相关性不高, 这是受学生动手能力的 限制、受学生专业能力的限制。但是, 近年来在一些新的 英文软件的培训中, 学生完全不同的表现是这样的观点无 法解释的。由于这些软件的操作界面与专业单词、专业知 识、实践能力相关性较低, 因此在软件界面操作的学习中 可以忽略学生专业能力的影响。而在面对这些新的英文软 件时, 传统阅读训练中通用英语取得较好成绩的学生, 并 不能明显快于其他学生掌握软件的操作界面。而在学生间 通过相互交流后, 所有学生掌握英文软件操作界面的水平 大幅提高。而且在使用母语汉语进行英文软件学习交流的 过程中, 即使通用英语程度较差的学生也能较好的参与交 流, 给出建设性的想法和意见。由此可见, 在真实环境中 忽略交流进行英语阅读能力培养是不现实的。

在真实性评价过程中为了模拟信息交流的过程可以组 织小组讨论, 在小组讨论后如有需要可以组织小组间的问 答, 在一些场景中可以由教师扮演一些咨询服务人员提供 咨询服务。在教学实践中, 可以发现这样的教学活动组织 难度较大, 特别是对于教师的组织能力有较高的要求。但 
是, 这些交流活动却更为接近真实环境学生可能面对的情

况。

\section{3 对于任务的设计}

在真实性环境的构建中, 学生阅读训练任务的设计也 是不可缺少的一部分。任务的设计主要是用于真实性评价 中对于学生阅读效果的评价。但是, 由于任务的差异, 所 需的信息也有不同, 这使得在真实性环境的构建中的确需 要相当注意。如果任务设计不恰当, 会直接影响构建真实 性环境的合理性。

在以往的英语能力训练中, 由于给出文字信息都在一 篇文章中, 虽然对不同的问题关注点不一样, 但是可以通 过重复阅读文章内容进行弥补, 但是在真实环境中一些情 况下这是不可能的。比如, 对于公交车、地铁等交通工具, 在上车前就需要明确车辆的行进方向, 这时候主要依赖车 辆站点的标示、以及上下车通道的差异进行保证。这时, 进行英语文本的阅读就有一定时效性的限制。

\section{4. 构建真实性环境值得注意的问题}

在教学实践中, 通过构建真实性环境为真实性评价建 立了基础, 这将较好的反映真实环境对学生英语阅读能力 的需求, 而在真实性环境构建中以下几个问题值得注意。

\section{1 真实性环境是语言环境的真实性}

在构建真实性环境中, 语言环境是最重要的环境, 而 一些物理环境的构建也是为了模拟真实性的环境。

如在对地铁站购票的模拟中, 通过视频可以给出地铁 站的情况, 通过教师模拟咨询人员可以进行问答, 通过宣 传手册、图片可以给出地铁参考运行情况……在教学过程 中, 这些都是构建真实性环境方式。以语言环境的真实性 作为评价标准, 这些方式并没有具体差异, 不能认为教师
模拟咨询人员场景不如视频真实而否定模拟咨询问答语言 环境的真实性。

\section{2 信息交流方式的可行性}

在进行基本阅读后, 按真实性环境允许学生进行一定 的信息交流, 但是值得注意的是选择这些交流方式的可行 性。比如进行咨询问答, 应该是在真实环境中可能出现咨 询人员的情况下进行, 而在阅读产品说明书时安排这样的 活动显然是不合理的。

\section{5. 小结}

针对大学生英语阅读能力培养的真实性评价, 讨论了 如何构建真实性环境, 这是进行真实性评价的基础, 将有 助于在教学中引入真实性评价提高大学生阅读能力的培养 质量。

\section{参考文献(References)}

[1] Li Yueping, Yang Tingjun. Research on relevance and trend of difficulty of traditional reading tests in CET-4. Journal of Guangxi normal university. Philosophy and social sciences edition, vol.49, pp. 124-128, 2013.

[2] Gan Ling, Jiang Yemei. Content validity of reading comprehension in college English final examinations. Journal of Guangxi normal university. philosophy and social sciences edition, vol.49, pp. 155-160, 2013

[3] Liu Yi. On application of authentic assessment to college English TBL with a case study. Journal of Chengdu college of education, vol.20, pp. 34-36, 2006

[4] Zhu Xiaohua. A study of authenticity of a reading achievement test. Journal of Weinan normal university. vol. 29, pp. 62-65, 2014.

[5] Wiggins, G. A true test: Toward more authentic and equitable assessment. Phi Delta Kappan, 1989, 70: 703-714 